\title{
Compreender como a espiritualidade e a religiosidade influenciam a experiência dos pacientes com câncer
}

\author{
Understanding how spirituality and religiosity \\ influence the experience of cancer patients
}

\author{
Rogevando Rodrigues Nunes* \\ Elaine Saraiva Feitosa** \\ Marcus Augusto Silva Ferreira*** \\ Raimunda Magalhães da Silva**** \\ Aline Veras Morais Brilhante***** \\ Ana Maria Fontenelle Catrib****** \\ Zélia Maria de Sousa Araújo Santos******* \\ Rosendo Freitas de Amorim********
}

Resumo: Objetivo. Analisar produçóes científicas disponíveis na literatura brasileira sobre a importância da espiritualidade e da religiosidade como suporte para pacientes oncológicos. Método. Trata-se de uma revisão integrativa da literatura realizada a partir de artigos publicados de 01.10.2009 a 30.09.2019 nas bases de dados da National Library of Medicine and the National Institutes of Health (PubMed), Biblioteca Virtual em Saúde (BVS), Literatura Latino Americana e do Caribe em Ciências da Saúde (LILACS) e da Scientific Eletronic Library Online (SCIELO). Resultados. A busca encontrou 13 artigos envolvendo 416 participantes. Conclusóes. A espiritualidade e a religiosidade são fatores essenciais para apoio do paciente oncológico. Há a necessidade de se promover uma integração dessa abordagem, envolvendo familiares e profissionais de saúde.

Palavras-chave: Espiritualidade. Religião. Câncer.

Abstract: Objective. To analyze scientific productions available in the Brazilian literature about the importance of spirituality and religiosity as support for cancer patients. Method. This is an integrative literature review conducted from articles published from 10.01.2009 to 09.30.2019 in the databases

\footnotetext{
* Professor do curso de graduação em Medicina da UNIFOR (Fortaleza-CE). Doutor em Saúde Coletiva (UNIFOR).ORCID: 0000-0002-6025-1480 - contato: rogevando@hotmail.com

** Preceptora do curso de graduação em Medicina da UNIFOR (Fortaleza-CE). Mestra em Saúde Pública (UECE). ORCID: 0000-0003-3006-4710 - contato: elainesfeitosa@gmail.com

*** Doutorando em Saúde Coletiva (UNIFOR, Fortaleza-CE). ORCID: 0000-0002-5300-5193 - contato: marcusasf@hotmail.com

**** Professora do PPG em Saúde Coletiva da UNIFOR (Fortaleza-CE). Doutora em Enfermagem (USP). ORCID: 0000-0001-5353-7520 - contato: rmsilva@unifor.br

***** Professora do PPG em Saúde Coletiva da UNIFOR (Fortaleza-CE). Doutora em Saúde Coletiva (UNIFOR). ORCID: 0000-0002-3925-4898 - contato: alineveras@unifor.br

****** Professora do PPG em Saúde Coletiva da UNIFOR (Fortaleza-CE). Doutora em Educação (UFBA). ORCID: 0000-0002-2088-0733 - contato: catrib@unifor.br

******* Professora do PPG em Saúde Coletiva da UNIFOR (Fortaleza-CE). Doutora em Enfermagem (UFC). ORCID: 0000-0002-5824-0723 - contato: zeliasantos@unifor.br

******** Professor do PPG em Saúde Coletiva da UNIFOR (Fortaleza-CE). Doutor em Sociologia (UFC). ORCID: 0000-0003-1498-8999 - contato: rosendo@unifor.br
} 
of the National Library of Medicine and the National Institutes of Health (PubMed), Virtual Health Library (VHL), Latin American and Caribbean Health Sciences Literature (LILACS) and the Scientific Electronic Library Online (SCIELO). Results. The search found 13 articles involving 416 participants. Conclusions. Spirituality and religiosity are essential factors for cancer patient support. There is a need to promote integration of this approach, involving family members and health professionals.

Keywords: Spirituality. Religion. Cancer.

\section{Introduçáo}

A espiritualidade envolve a busca da transcendência, cultivando a fé de maneira mais ampla. Enquanto a religiosidade está ancorada em princípios mais específicos, como ritos, crenças e dogmas (Marques, 2010, p. 139). A religiosidade oportuniza uma reflexão do indivíduo sobre sua vida e o motivo de sua existência além do mundo concreto. Para Tavares et al., (2018, p. 1098), fé e espiritualidade podem atuar estrategicamente, proporcionando uma melhor convivência com determinado problema. Embora frequentemente associadas, espiritualidade e religiosidade não sáo necessariamente interdependentes e uma pessoa pode se desenvolver espiritualmente sem recorrer a um sistema religioso ou metafísico (Solomon, 2003, p. 18; Pessini, 2010, p. 106). Religiosidade e doença possuem um elo consistente, pois foi verificado que práticas religiosas podem proporcionar aspectos positivos ou negativos no que concerne à saúde física ou mental dos adeptos (Thiengo et al., 2019).

O modelo biomédico clássico leva em conta somente os aspectos biológicos da doença, enquanto o modelo biopsicossocial considera a visão integral do doente, interessando-se tanto pela dimensão física quanto por aspectos psicológicos e sociais (Aragão, 2016). Nesse contexto, a compreensão do papel da espiritualidade e da religiosidade na experiência de doenças marcadas por um estigma - como o câncer - contribui para uma prática profissional centrada na integralidade da pessoa e não, somente, na doença.

De acordo com o INCA (2018, pp. 13, 30 e 31), "câncer" é uma designação genérica empregada para um grupo de mais de 100 doenças diferentes que têm em comum a proliferação celular desordenada, sendo esperados 600 mil novos casos desta enfermidade, no Brasil, em 2019. Os tipos de câncer mais comuns registrados pelo INCA afetam os seguintes órgáos: mamas, próstata, pele, pulmóes, estômago, cólon, reto, fígado, colo do útero, cavidade oral, traqueia e tireoide.

Embora diferentes em etiologia, gravidade dos sintomas, progressáo e prognóstico, as diferentes enfermidades agrupadas sob o estereótipo do "câncer" são socialmente estigmatizadas, resultando em angústia psicológica e ideação - às vezes verossímil, outras vezes falaciosas - da morte iminente (Johnson, 2019, pp. 324-325). Vale ressaltar que a maioria dos cânceres tem o envelhecimento como fator de risco, o que implica a previsão de um aumento nos diagnósticos à medida que a expectativa de vida da populaçáo se expande (Goldman; Schafer, 2018, p. 1242). O Brasil está vivenciando esse processo de aumento da populaçáo idosa (IBGE, 2018, p. 30).

O aumento progressivo da prevalência de doenças neoplásicas e a aproximação epistemológica do campo da saúde com correntes da antropologia (Marques, 2010, pp. 135-151) 
colocaram em evidência a importância da espiritualidade e religiosidade na melhoria do bem-estar dos pacientes com câncer (Xing et al., 2018, p. 10; Vitorino et al., 2018, p. 3). Os níveis de espiritualidade e esperança estáo diretamente correlacionados (Souza et al., 2017, p. 7). No entanto, ainda existem lacunas entre esses temas e serviços de assistência (Evangelista et al., 2016, pp. 599). A maior divulgaçáo de estudos sobre a importância da espiritualidade e da religiosidade é um ponto necessário para reduzir a abordagem fragmentada do corpo humano, em que o físico e o espiritual são desmembrados e individualizados. Pacientes que recorrem à religiosidade mudam seus estilos de vida para práticas mais saudáveis, geralmente melhorando a sensação de bem-estar (Lago; Abdala; Meira, 2017, p. 2609). É fundamental promover uma prática de cuidado integral, centrado na pessoa.

Nesse contexto, surgiu a questão: como a espiritualidade e a religiosidade podem interferir positivamente na evolução dos pacientes com câncer? Sendo a idade avançada um fator de risco para câncer, é esperado que os adultos e idosos sejam os grupos mais prevalentes nos estudos já realizados, sendo o objeto principal desta pesquisa. Com base nessa questáo norteadora, este estudo pretende compreender como a espiritualidade e a religiosidade influenciam a experiência dos pacientes com câncer no Brasil.

\section{Metodologia}

Trata-se de uma revisão integrativa da literatura, um método de pesquisa que analisa artigos de várias metodologias, seguindo uma padronização pré-estabelecida para buscar novos conhecimentos sobre determinado assunto (Mendes; Silveira; Galvão, 2008, pp. 758-764; Souza; Silva; Carvalho, 2010, pp. 102-106). Este trabalho foi realizado durante os meses de abril e maio de 2020 por oito pesquisadores.

\section{Figura 1 - Fluxograma dos artigos excluídos e selecionados.}

Foram encontrados 40 artigos

$\sqrt{1}$

12 artigos foram excluídos por repetição

\section{$\sqrt{ }$}

15 artigos foram excluídos porque não abordavam

o tema da pesquisa nos resumos ou nos textos

Restaram 13 artigos que foram analisados (416 participantes)

Fonte: elaboração dos autores (2020).

Foi efetuada uma pesquisa em artigos publicados de 01.10.2009 a 30.09.2019 nas bases de dados PubMed, BVS, LILACS e SCIELO. Foram selecionados artigos em 
português, citáveis, textos completos gratuitos, disponíveis e provenientes do Brasil. Foi realizada uma busca nos Descritores em Ciências da Saúde (DeCS/MeSH) para encontrar os artigos. As palavras norteadoras da pesquisa foram: espiritualidade, religiosidade, câncer. Empregando-se os operadores booleanos AND e OR, a expressão utilizada na pesquisa foi: "Espiritualidade OR religiosidade AND câncer". Artigos repetidos e aqueles que apresentaram distanciamento do tema proposto foram excluídos. De acordo com a Figura 1, das 40 publicaçôes encontradas inicialmente, após excluir as repetidas e as que não abordavam o tema da pesquisa durante a leitura dos resumos ou dos textos, restaram 13 artigos. A temporalidade dos últimos 10 anos foi escolhida por representar um período relativamente longo, mas atual, porque já está inserida no século XXI. A língua portuguesa foi selecionada para dar preferência a estudos realizados no Brasil.

\section{Resultados}

Os artigos selecionados foram lidos na íntegra, analisados e sintetizados nos Quadros 1 e 2 . O número total de participantes foi 416 . As publicaçôes ocorreram nos seguintes períodos: 2019, dois artigos; 2017, dois artigos; 2016, um artigo; 2015, dois artigos; 2014, dois artigos; 2012, um artigo; 2010, um artigo e 2009, dois artigos. Alguns desses artigos estavam disponíveis, além de no idioma português, também em outros idiomas. Três adotaram a metodologia quantitativa, envolvendo 157 participantes, e dez empregaram metodologia qualitativa, estudando 259 participantes. Sete artigos foram publicados nos últimos cinco anos. Os artigos foram publicados em revistas de enfermagem: 7, revistas de psicologia: 3 e revistas multidisciplinares: 3 .

Dez artigos discriminaram o gênero dos participantes (365 participantes), sendo que 96 eram do sexo masculino e 269 eram do sexo feminino. Oito artigos (113 participantes) especificaram a religiáo adotada pelos participantes, sendo 63 católicos, 37 evangélicos, 6 protestantes, 5 sem religião e 2 espíritas. Em relação aos tipos de câncer dos participantes, dez artigos fizeram alusáo a este dado, sendo que 224 eram câncer de mama e o restante envolvia pacientes que eram portadores de um dos seguintes: mama, próstata, estômago e intestinos, tireoide, hematológico, cabeça e pescoço, fígado, pênis, pulmão, útero, ovário e testículo.

A partir da leitura dos artigos, emergiram duas categorias temáticas: a) Enfrentamento da doença; b) Esperança de cura e compreensão da finitude.

\section{Discussáo}

\section{Enfrentamento da doença}

Após a má notícia do diagnóstico do câncer, há estresse, angústia, sofrimento, desesperança, sensação de impotência e de esvaziamento motivacional. Há busca de um sentido para a vida após a descoberta do câncer (Reis; Farias; Quintana, 2017, p. 109). Nesse momento, o paciente precisa de uma base de apoio que ofereça esperança, conforto e qualidade de vida para que haja enfrentamento e aceitação da doença. Esse 
apoio, geralmente, vem de familiares e amigos, além da maior aceitação e desenvolvimento do lado espiritual e/ou religioso (Fernandes; Carvalho; Ferreira, 2019, p. 79).

Os pacientes com câncer frequentemente enfrentam um vazio de sentido para a vida, seja por movimentos externos, como o afastamento e a falta de olhar da equipe de atendimento, amigos e familiares, bem como por dimensóes subjetivas e singulares relacionadas à vivência da doença (Reis; Farias; Quintana, 2017, pp. 107-108). Os pacientes buscam construir um sentido para a vida, predominantemente através da religiosidade, o que lhes oferece possibilidades de compreensão, apoio, conforto, sentimento de controle, entre outros construtos de significado para a doença (Ribeiro; Campos; Anjos, 2019, pp. 854, 856). Deus é colocado como guardião da esperança de cura, identificado como protetor e como responsável pela situação que os pacientes estão enfrentando. Entretanto, às vezes, a religiosidade não se mostra suficiente para o trabalho de construção de significados para o adoecimento (Reis; Farias; Quintana, 2017, p. 116).

De acordo com Boff, ao se referir às autoridades da Igreja,

[...] a espiritualidade como o essencial, independentemente da crença religiosa, deve ser incentivada, nada impedindo que a pessoa a vivencie em uma crença específica. Evitando-se uma fé imatura e a subserviência infantil às autoridades (Boff, 2001, p. 19).

Vários depoimentos registrados por Miranda; Lanna e Felippe (2015, p. 881) evidenciam que o câncer desperta, no paciente, a busca pela espiritualidade. Entre os pacientes, prevalece a religiosidade e a espiritualidade vividas, aparentemente, em harmonia. Sugere-se que a intensidade e maturidade da população vivenciando religiosidade e/ou espiritualidade no Brasil pode ter alcançado essa harmonia, atuando como um antídoto para o que Leonardo Boff alerta como automação da religiáo, afirmando o seguinte:

Quando a religião esquece a espiritualidade, pode ser automatizada articulando poderes religiosos com outros poderes. Ao se institucionalizarem na forma de poder, seja sagrado, social ou cultural, as religióes perdem a fonte que as mantém vivas - a espiritualidade (Boff 2001, p. 19).

A forte tradição religiosa brasileira, com predominância da fé cristã, é significativa. É relevante a comparação da fala de Leonardo Boff com a realidade identificada.

Existem iniciativas legais que podem ser utilizadas em situações específicas de assistência religiosa e espiritual, como Lei No 9.982/2000. Essa lei foi regulamentada pelo Decreto No 30.582/2009, que estabelece diretrizes para serviços baseados na fé em instituições públicas e privadas para prestar assistência religiosa aos pacientes, desde que eles e/ou seus familiares estejam de acordo.

É necessário que a questão esteja além da natureza legal e aspire a humanização com o envolvimento da tríade composta por: pacientes, profissionais de saúde e família, na construção de uma nova cultura em relação ao problema gerado pela doença oncológica. A religiosidade promove uma troca de energia positiva que funciona reduzindo a ansiedade, o medo e a insegurança (Carvalho et al., 2014, pp. 684 e 688). A espiritualidade parece emergir como um componente gerador de esperança, ajudando 
a enfrentar os desafios relacionados ao câncer e a busca de sentido na vida. De acordo com Souza et al (2015, p. 512), a espiritualidade, a crença e a fé são consideradas essenciais para o conforto e o enfrentamento do estresse gerado pelo câncer. A prática da espiritualidade, incluindo crenças, valores e rituais durante o processo de adoecimento, pode trazer os seguintes benefícios: aumentar sentimentos de força, tranquilidade e confiança; promover autocuidado, ajudando na recuperação e restaurando a saúde; promover apoio espiritual e emocional da comunidade; contribuir para sentimentos de pertencimento a uma comunidade; dar significado à vida, amigos e família (Souza et al., 2015, p. 513).

A religião aparece como uma estratégia de enfrentamento dos desafios e da própria continuidade da vida de pacientes penectomizados. A espiritualidade é fundamental para a aceitação da doença e a adesão ao tratamento. As entidades divinas são exaltadas como responsáveis por guiá-los em suas decisões cotidianas, como a realização de cirurgias. A religião é vista como uma estratégia para lidar com a doença e pela a continuidade da vida (Araújo et al., 2014, pp. 470-471).

A esperança das mulheres com câncer de mama, sob quimioterapia, foi avaliada desde o início e melhorou, significativamente, ao final deste tratamento. Pacientes que relataram dor no início do tratamento tiveram uma pontuação de esperança diminuída. Entre as variáveis analisadas, a dor foi o único preditor de esperança. O quadro álgico evidencia uma chance de reduzir a esperança em 2,199 vezes (Balsanelli; Grossi, 2016, p. 902). Na presença de doenças, há uma relação positiva entre religiosidade e espiritualidade com melhora na qualidade de vida, melhora resposta imunológica e há redução no tempo de internação e de quadros depressivos (Tavares et al., 2018, p. 1100).

\section{Esperança de cura e compreensão da finitude}

O apoio da religiosidade e/ou da espiritualidade pode ser uma estratégia eficaz na luta contra o câncer. Muitos pacientes relatam o significado positivo do apoio religioso recebido, pois a fé fornece uma maneira de pensar construtiva (Freire et al., 2017, pp. 361). A ajuda emocional proveniente da espiritualidade incentiva os pacientes a serem otimistas e esperançosos, buscando conviver com familiares e amigos, tentando levar uma vida normal, dentro de suas possibilidades.

É necessário que os profissionais de saúde utilizem mais o artifício da espiritualidade e da religiosidade durante a comunicação com pacientes oncológicos. Isso pode levar a uma maior resiliência por parte dos doentes e uma alta taxa de adesáo ao tratamento proposto, contribuindo para uma melhor qualidade de vida. O bem-estar do paciente passa necessariamente pelo seu estado psicológico, físico e social. A definição de saúde da Organização Mundial da Saúde refere-se ao completo bem-estar físico, mental e social. De acordo com essa definição, a depender de sua percepçáo da vida, um paciente com câncer pode sentir-se mais saudável que outro, que apresenta problemas físicos, mentais, emocionais, psicológicos e/ou sociais.

O suporte vinculado à religiosidade e à espiritualidade precisa ser aprofundado, principalmente porque são pessoas que correm o risco de perder ou danificar, em 
pouco tempo, o que há de mais importante: a vida, seja por severas limitações em sua qualidade ou consequente à sua própria finitude.

A religiosidade colabora para o preenchimento do vazio que surge diante de uma doença grave como o câncer. Quando o sentimento de finitude não é suficientemente compreendido e aceito, outras estratégias serão necessárias para que o paciente viva com a possibilidade de morte, porque muitas vezes são doentes terminais (Reis; Farias; Quintana, 2017, p. 116). Para vários pacientes, a vida continua com boa qualidade. A parte espiritual assume uma importância salutar, porque um paciente deprimido e sem esperança perde o entusiasmo pela vida. Essas pessoas podem morrer décadas após o diagnóstico, inclusive por fatores não relacionados ao câncer.

A doença atinge o corpo e a mente. É impossível dissociar a doença física de uma pessoa de suas emoçóes (doença mental), representadas por angústia, medo, revolta e um senso de finitude. A espiritualidade preenche uma lacuna na superação ou minimização das consequências geradas pelo processo da doença, principalmente no que está relacionado à esfera psicológica e emocional (Souza et al., 2015, pp. 509-514).

A espiritualidade, independentemente da crença religiosa do paciente, atua como um protetor em tempos de provaçáo diante de uma doença potencialmente grave ou incurável. Isso vai além do efeito placebo, pois ainda há muito a desvendar sobre fatores subjetivos no processo de cura ou na aceitação de uma doença.

\section{Consideraçóes finais}

Apesar da estreita relação entre sistemas de crenças e sofrimento na experiência de diferentes tipos de câncer, as práticas clínicas hegemônicas ainda tendem a ver o paciente com câncer de maneira cartesiana, compartimentalizada. Estratégias precisam ser construídas para auxiliar o paciente a lidar com a ansiedade relacionada à doença, especialmente quando existe a possibilidade de morte. A relação entre esperança, espiritualidade, religiosidade e os benefícios produzidos para a melhora ou aceitação do paciente é evidente. Desse modo, infere-se que a espiritualidade e a religiosidade são capazes de trazer certas explicaçóes e suportes diante do esvaziamento dos sentidos causados pela doença oncológica.

A espiritualidade e a religiosidade são fatores relevante na superação do câncer, incluindo pacientes internados, ou seja, pessoas sujeitas a um ambiente com muitas limitaçóes e riscos. A espiritualidade e/ou da religiosidade proporcionam esperança, que é geradora de vários benefícios, como: força, melhora do humor, tranquilidade e confiança. Uma maneira construtiva e confiante de pensar é mostrada como um ganho proporcionado pela espiritualidade. Esse otimismo é essencial para que a vida cotidiana seja vivida de maneira agradável.

Incentivar o cultivo da espiritualidade e da religiosidade é uma estratégia que mostra bons resultados durante a avaliação de estudos realizados e publicados na literatura. Essa prática deve ser sistematizada para pessoas que enfrentam situaçóes de risco de morte. O cultivo da espiritualidade pode ser efetivado através de palestras com filósofos e religiosos sobre o sentido e o propósito da vida. Já a religiosidade poderia ser 
desenvolvida através da participaçáo em grupos religiosos, incentivo a envolvimento com crenças e rituais religiosos específicos. Esses pacientes se sentem mais energizados quando abraçam a fé e a esperança, valores diretamente relacionados à espiritualidade e à religiosidade. A maneira como uma pessoa se percebe no mundo está relacionada à sua dimensão espiritual. É salutar que haja maior disseminação da espiritualidade e da religiosidade na formação dos profissionais de saúde, para que eles possam prestar assistência à saúde de forma mais integral.

É evidente que o papel dos profissionais de saúde: médicos, enfermeiros, terapeutas ocupacionais, entre outros, é fundamental para a obtençáo de resultados positivos na superação desse desafio. Quanto mais sensibilizados esses profissionais estiverem para integrar a espiritualidade e a religiosidade à recuperação do paciente, melhores resultados poderão ser alcançados durante o tratamento, seja curando ou aceitando a condição, evitando ou minimizando sentimentos destrutivos de desespero ou negaçáo prolongada. Destacamos também o papel da família e da comunidade na qual o paciente está inserido. $\mathrm{O}$ apoio espiritual das pessoas mais próximas, sejam familiares, amigos, vizinhos ou mesmo pacientes em tratamento semelhante, pode construir uma rede de proteção e força que pode ajudar o indivíduo nesse momento de fragilidade.

Considerando as características transcendentais da sociedade brasileira e o surgimento de maior número de casos de câncer nos próximos anos, a religiosidade e a espiritualidade devem ser fortalecidas e fomentadas no âmbito de políticas públicas e agentes de saúde como forma de humanizar os tratamentos, transformando a realidade em favor da populaçáo afetada.

\section{Referências}

ARAGÃO, Soraya Rodrigues de. O Modelo Biomédico X o Modelo Biopsicossocial na Explicação da Depressão. Psicologado, [S.l.]. (2016). Disponível em https:// psicologado.com.br/psicopatologia/saude-mental/o-modelo-biomedico-x-o-modelobiopsicossocial-na-explicacao-da-depressao. Acesso em 28 abril de 2020.

ARAÚJO, Jeferson Santos; XAVIER, Érika de Cássia Lima; CONCEIÇÃO, Vander Monteiro; SILVA, Silvio Eder Dias; RODRIGUES, Ivaneide Leal Ataide; VASCONCELOS, Esleane Vilela. Os atos representacionais do falo no cotidiano do homem penectomizado: a amputação, religiosidade e a família. Revista de Pesquisa: Cuidado é Fundamental Online, v. 6, n. 2, pp. 462-473, abr/jun 2014.

BALSANELLI, Alessandra Cristina Sartore; GROSSI, Sonia Aurora Alves.

Predictors of hope among women with breast cancer during chemotherapy. Revista da Escola de Enfermagem da USP, v. 50, n. 6, pp. 898-904, nov/dec 2016.

BOFF, Leonardo. Espiritualidade - um caminho de transformação - Rio de Janeiro: Sextante, p. 19. 2001.

CARVALHO, Camila Csizmar; CHAVES, Erika de Cássia Lopes; IUNES, Denise Hollanda; SIMÃO, Talita Prado; GRASSELLI, Cristiane da Silva Marciano; BRAGA, Cristiane Giffoni. Effectiveness of prayer in reducing anxiety in cancer patients. Revista da Escola de Enfermagem da USP, v. 48, n. 4, pp. 684-690, 2014. 
EVANGELISTA, Carla Braz; LOPES, Maria Emília Limeira; COSTA, Solange Fátima Geraldo; BATISTA, Patrícia Serpa de Souza; BATISTA, Jaqueline Brito Vidal; OLIVEIRA, Amanda Maritsa de Magalhães. Cuidados paliativos e espiritualidade: revisão integrativa da literatura. Revista Brasileira de Enfermagem, v. 69, n. 3, pp. 591-601, mai/jun 2016.

FERNANDES, Maria Julyê Mota; CARVALHO, Gabriela Borges; FERREIRA, Cintia Bragheto. Repercussóes do diagnóstico de câncer para homens e mulheres: um estudo comparativo. Revista da SPAGESP, v. 20, n. 2, pp. 68-83, 2019.

FREIRE, Maria Eliane Moreira; VASCONCELOS, Monica Ferreira; SILVA, Terezinha Nunes; OLIVEIRA, Karla De Lima. Spiritual and religious assistance to cancer patients in the hospital context. Revista de Pesquisa: Cuidado é Fundamental Online, v. 9, n. 2, pp. 356-362, 2017.

GOLDMAN, Lee; SCHAFER, Andrew I. Goldman-Cecil Medicina. 25. ed. Rio de Janeiro: Elsevier, 2018.

GUERRERO, Giselle Patrícia; ZAGO, Márcia Maria Fontão; SAWADA, Namie Okino; PINTO, Maria Helena. Relação entre espiritualidade e câncer: perspectiva do paciente. Revista Brasileira de Enfermagem, v. 64, n. 1, pp. 53-59, jan/fev 2011.

INSTITUTO BRASILEIRO DE GEOGRAFIA E ESTATÍSTICA - IBGE. Pesquisa Nacional por Amostra de Domicílio (Características gerais dos domicílios e dos moradores 2017). 2018. Disponível em: http://biblioteca.ibge.gov.br/visualizacao/ livros/liv101566_informativo.pdf. Acesso em: 23/05/2020.

INSTITUTO NACIONAL DO CÂNCER - INCA. Brasil. ABC do câncer: abordagens básicas para o controle do câncer. 4. ed. Rio de Janeiro: INCA, 2018, 111 pp. Disponível em: https://www.inca.gov.br/sites/ufu.sti.inca.local/files//media/ document//livro-abc-4-edicao.pdf. Acesso em 26 de maio de 2020.

JOHNSON, Lee Ann; SCHREIER, Ann M; SWANSON, Melvin; MOYE, Janet P; RIDNER, Sheila. Stigma and Quality of Life in Patients With Advanced Lung Cancer. Oncology Nursing Forum, v. 46, n. 3, pp. 318-328, may 2019.

LAGO, Raquel Conceição; ABDALA, Gina Andrade; MEIRA, Maria Dyrce Dias. RELIGIOSITY AND LIFESTYLE OF HYPERTENSIVE PATIENTS IN THE FAMILY HEALTH STRATEGY. Journal of Nursing UFPE on line, v. 11 (suppl. 6), pp. 2604-2611, june 2017.

MARQUES, Luciana Fernandes. O conceito da espiritualidade e sua interface com a religiosidade e a Psicologia Positiva. Psicodebate, v. 10, pp. 135-151, 2010.

MENDES, Karina Dal Sasso; SILVEIRA, Renata Cristina de Campos Pereira; GALVÃO, Cristina Maria. Revisão Integrativa: método de pesquisa para a incorporação de evidências na saúde e na enfermagem. Texto \& Contexto Enfermagem 2008, v. 17, n. 4, pp. 758-764, out/dez 2008.

MENEZES, Natália Nogueira Teixeira; SCHULZ, Vera Lucia; PERES, Rodrigo Sanches. Impacto psicológico do diagnóstico do câncer de mama: um estudo a partir 
dos relatos de pacientes em um grupo de apoio. Estudos de Psicologia, v. 17, n. 2, pp. 233-240, mai/ago 2012.

MIRANDA, Sirlene Lopes; LANNA, Maria dos Anjos Lara; FELIPPE, Wanderley Chieppe. Espiritualidade, Depressão e Qualidade de Vida no Enfrentamento do Câncer: Estudo Exploratório. Psicologia: Ciência e Profissão, v. 35, n. 3, pp. 870$885,2015$.

PESSINI, Leo. Espiritualidade e arte de cuidar: o sentido da fé para a saúde. Sáo Paulo: Paulinas/Centro Universitário São Camilo, p. 106, 2010.

REIS, Cristine Gabrielle da Costa; FARIAS, Camila Peixoto; QUINTANA, Alberto Manuel. O Vazio de Sentido: Suporte da Religiosidade para Pacientes com Câncer Avançado. Psicologia: Ciência e Profissão, v. 37, n. 1, pp. 106-118, jan/mar 2017.

RIBEIRO, Gabriella Santos; CAMPOS, Cristiane Soares; ANJOS, Anna Cláudia Yokoyama dos. Spirituality and religion as resources for confronting breast cancer. Revista de Pesquisa: Cuidado é Fundamental Online, [S.I.], v. 11, n. 4, pp. 849-856, jul/set 2019.

SANCHEZ, Keila de Oliveira Lisboa; FERREIRA, Noeli Marchioro Liston Andrade; DUPAS, Giselle; COSTA, Giselle. Apoio social à família do paciente com câncer: identificando caminhos e direçóes. Revista Brasileira de Enfermagem, v. 63, n. 2, pp. 290-299, mar/abr 2010.

SOLOMON, Robert C. Espiritualidade para céticos: paixão, verdade cósmica e racionalidade no século XXI. Rio de Janeiro: Civilização Brasileira, p. 18, 2003.

SOUZA, Marcela Tavares; SILVA, Michelly Dias; CARVALHO, Rachel. Integrative review: what is it? How to do it? Einstein, v. 8(1 Pt 1), pp.102-106, 2010.

SOUZA, Verônica de Moura; FRIZZO, Heloísa Cristina Figueiredo; PAIVA, Michelle Helena Pereira; BOUSSO, Regina Szylit; SANTOS, Álvaro da Silva. Spirituality, religion and personal beliefs of adolescents with cancer. Revista Brasileira de Enfermagem, v. 68, n. 5, pp. 509-514, sept-oct 2015.

SOUZA, Érica Nestor; OLIVEIRA, Nathalia Alves; LUCHESI, Bruna Moretti; GRATÃO, Aline Cristina Martins; ORLANDI, Fabiana de Souza; PAVARINI, Sofia Cristina Iost. Relationship between hope and spirituality of elderly caregivers. Texto \& Contexto - Enfermagem, v. 26, n. 3:e6780015, pp. 1-8, aug 2017.

SPADACIO, Cristiane; BARROS, Nelson Filice. Terapêuticas convencionais e não convencionais no tratamento do câncer: os sentidos das práticas religiosas. Interface - Comunicação, Saúde, Educação, v. 13, n. 30, pp. 45-52, set 2009.

TAVARES, Marilei de Melo; GOMES, Antonio Marcos Tosoli; BARBOSA, Diogo Jacintho; ROCHA, Julio Cesar Cruz Collares; BERNARDES, Margarida Maria Rocha; THIENGO, Priscila Cristina da Silva. Spirituality and religiosity in the daily routine of hospital nursing. Journal of Nursing UFPE on line, v. 12, n. 4, pp. 1097$1102,2018$. 
THIENGO, Priscila Cristina da Silva; GOMES, Antonio Marcos Tosoli; MERCÊS, Magno Conceição das; COUTO, Pablo Luiz Santos; FRANÇA, Luiz Carlos Moraes; SILVA, Alba Nunes da. SPIRITUALITY AND RELIGIOSITYY IN HEALTH CARE: AN INTEGRATIVE REVIEW. Cogitare Enfermagem, v. 24: e58692, 2019.

VITORINO, Luciano Magalhães; LUCCHETTI, Giancarlo; LEÃO, Frederico Camelo; VALLADA, Homero \& PERES Mario Fernando Prieto. The association between spirituality and religiousness and mental health. SCIentIFIC RepoRTs, v. 8:17233, pp. 1-9, 2018.

XING, Lu; GUO, Xiujing; BAI, Lu; QIAN, Jiahui; CHEN, Jing. Are spiritual interventions beneficial to patients with cancer? A meta-analysis of randomized controlled trials following PRISMA. Medicine, v. 97, n. 35:e11948, pp. 1-11, aug 2018 .

Recebido: 5 de dezembro de 2019.

Aprovado: 13 de maio de 2020. 


\section{Quadro 1 - Estudos sobre espiritualidade e/ou religiosidade, no câncer, conforme autor/ano/título, número de participantes(n) e objetivo}

\section{Autor/ano/título}

Ribeiro; Campos; Anjos, 2019. Espiritualidade

1 e religiáo como recursos para o enfrentamento do câncer de mama

Fernandes; Carvalho; Ferreira, 2019.

2 Repercussóes do diagnóstico de câncer para homens e mulheres: um estudo comparativo

Reis; Farias; Quintana, 2017. O vazio

3 de sentido: suporte da religiosidade para pacientes com câncer avançado

Freire et al., 2017. Assistência espiritual e

4 religiosa a pacientes com câncer no contexto hospitalar

Balsanelli; Grossi, 2016. Fatores preditores

5 da esperança entre mulheres com câncer de mama durante o tratamento quimioterápico

Miranda; Lanna; Felippe, 2015.

6 Espiritualidade, depressão e qualidade de vida no enfrentamento do câncer: estudo exploratório

Souza et al., 2015. Espiritualidade,

7 religiosidade e crenças pessoais de adolescentes com câncer

Carvalho et al., 2014. A efetividade da prece

8 na redução da ansiedade em pacientes com câncer

Araújo et al., 2014. Os atos representacionais do

9 falo no cotidiano do homem penectomizado: a amputação, religiosidade e a família

Menezes; Schulz; Peres, 2012. Impacto

10 psicológico do diagnóstico do câncer de mama: um estudo a partir dos relatos de pacientes em um grupo de apoio

Guerrero et al., 2011. Relação entre

11 espiritualidade e câncer: perspectiva do paciente

Sanchez et al., 2010. Apoio social à família do

12 paciente com câncer: identificando caminhos e direçóes

Spadacio; Barros, 2009. Terapêuticas

13 convencionais e náo convencionais no tratamento do câncer: os sentidos das práticas religiosas n Objetivo

Descrever e compreender como uma paciente

1 com câncer de mama utiliza a religiosidade e a espiritualidade para enfrentar essa doença

Identificar, descrever e comparar as

16 repercussóes do diagnóstico de câncer para homens e mulheres

Compreender quais os sentidos que os

12 pacientes constroem para a vivência do adoecimento grave

Investigar como os pacientes oncológicos

25 concebem apoio religioso e espiritual no contexto hospitalar

Identificar os fatores preditores da esperança

122 nas pacientes com câncer de mama sob tratamento quimioterápico

Investigar inter-relaçóes entre bem-estar espiritual, depressão e qualidade de vida

15 durante o enfrentamento do câncer por pacientes da Casa de Apoio aos Pacientes com Câncer

Analisar a percepçáo dos adolescentes com

09 câncer sobre espiritualidade, religiấo e crenças pessoais em suas vidas e uma possível estratégia para lidar com a doença

Avaliar o efeito da prece sobre a ansiedade

20 de pacientes com câncer sob tratamento quimioterápico

Identificar e analisar as representaçóes sociais

30 de pacientes após penectomia radical devido ao câncer de pênis e suas implicaçôes para a saúde

Analisar o impacto psicológico do diagnóstico do câncer de mama a partir dos

93 relatos apresentados espontaneamente por mulheres acometidas pela doença durante as sessóes de um grupo de apoio

Compreender a relação entre a espiritualidade

14 e o câncer, na perspectiva dos pacientes oncológicos

Identificar e analisar artigos publicados

51 sobre apoio social e familiar do doente com câncer, sintetizando quais aspectos estão sendo estudados pelos pesquisadores

Apreender o sentido do uso de alternativas

08 complementares por pacientes em tratamento do câncer

Fonte: elaboração dos autores, com base na revisão integrativa (2020). 


\section{Quadro 2 - Estudos sobre espiritualidade em oncologia, conforme o método/ idade e resultados}

\begin{tabular}{|c|c|c|}
\hline & Método/idade & Resultados \\
\hline 1 & $\begin{array}{l}\text { Qualitativo/54 } \\
\text { anos }\end{array}$ & $\begin{array}{l}\text { A participante descreveu a fé e a religiáo como fontes de apoio. A religiosidade trouxe } \\
\text { mais ânimo, forças e vontade de viver. A fé ocupou papel de destaque no enfrentamento } \\
\text { do câncer }\end{array}$ \\
\hline 2 & $\begin{array}{l}\text { Qualitativo/ } \\
\text { não especifica }\end{array}$ & $\begin{array}{l}\text { Estudo descritivo em que a religiosidade e/ou espiritualidade estão relacionadas a } \\
\text { diminuição da ansiedade, tristeza, sofrimento e raiva. Estáo vinculadas com esperança } \\
\text { de cura e melhor qualidade de vida. Os grupos de participantes, homens e mulheres, } \\
\text { apresentaram reaçóes similares }\end{array}$ \\
\hline 3 & $\begin{array}{l}\text { Qualitativo/não } \\
\text { especificada }\end{array}$ & $\begin{array}{l}\text { Os participantes buscavam um sentido para a vida, através da religiosidade. Deus representa } \\
\text { a esperança de cura, situado como protetor e como responsável pelo adoecimento que } \\
\text { os pacientes estão vivenciando. A religiosidade é capaz de lhes trazer certas explicaçôes } \\
\text { e suportes diante do esvaziamento dos sentidos provocado pela doença. Algumas vezes, } \\
\text { a espiritualidade e a religiosidade podem ser insuficientes como única base de apoio } \\
\text { para os pacientes oncológicos }\end{array}$ \\
\hline
\end{tabular}

\begin{tabular}{cl}
\hline Qualitativo/ & Os pacientes concebem um apoio espiritual e religioso proveniente dos profissionais de \\
acima de 18 anos & saúde da instituiçáo, de voluntários, terceiros ou transeuntes
\end{tabular}

\begin{tabular}{|c|c|c|}
\hline 5 & $\begin{array}{l}\text { Quantitativo/ } \\
\text { média de } \\
50 \text { anos }\end{array}$ & $\begin{array}{l}\text { A esperança foi avaliada nas mulheres com câncer de mama, no decorrer da quimioterapia, } \\
\text { pela escala de Herth. A dor foi identificada como sendo o único fator preditor da } \\
\text { esperança: pacientes com mais dor no início da terapia tiveram escore de esperança } \\
\text { diminuído no final do tratamento. A religiosidade, avaliada pela escala de Duke, nâo } \\
\text { apresentou correlaçáao significativas com a esperança }\end{array}$ \\
\hline 6 & $\begin{array}{c}\text { Quantitativo/41 } \\
\text { a } 60 \text { anos }\end{array}$ & $\begin{array}{l}\text { Toda população estudada apresentou bem-estar espiritual e religioso moderado, o que } \\
\text { sugere que o câncer favorece a busca pela espiritualidade e religiosidade como mecanismos } \\
\text { para enfrentar o sofrimento. A religiosidade e a espiritualidade se apresentam como fontes } \\
\text { de esperança para a cura e como uma maneira de ressignificar o sentido da vida e da morte }\end{array}$ \\
\hline
\end{tabular}

A maioria dos adolescentes reconheceu a importância da espiritualidade no enfrentamento da doença, especialmente a partir de rituais religiosos e apoio da comunidade. Segundo eles, a prática da espiritualidade, incluindo crenças, valores e rituais distintos, durante o

Qualitativo/12
a 18 anos processo de adoecimento, pode trazer os seguintes benefícios: aumentar os sentimentos de força, tranquilidade e confiança; promover o autocuidado, auxiliando a recuperação e o restabelecimento; promover o apoio emocional; contribuir para os sentimentos de pertencimento a uma comunidade; dar significado à vida, aos amigos e à família. Espiritualidade, crença e fé são muito importantes para vencer o estresse gerado pela doença oncológica

8 Quantitativo/40 A fé e a espiritualidade são estratégias eficientes na redução da ansiedade do paciente a 70 anos $(90 \%)$ com câncer sob quimioterapia

O câncer de pênis é uma doença bastante temida entre os homens, detentora de um

9 Qualitativo/20 poder social, biológico e simbólico que controla o indivíduo. A espiritualidade e a a 79 anos religiosidade foram vistas como fatores importantes de enfrentamento, aceitaçáo da doença e adesão ao tratamento

O diagnóstico do câncer proporcionou, entre uma parcela expressiva das participantes,

10 Qualitativo/50 um impacto psicológico importante. A notícia desencadeou experiências de surpresa a 70 anos e tensão, demonstraçóes de aceitação e força, motivando tentativas de explicação, redefinindo relacionamentos e intensificando a busca pela religiosidade

\begin{tabular}{ccc}
\hline Qualitativo/ & Há uma busca da espiritualidade como uma maneira de enfrentamento do câncer e \\
acima de 18 anos & para minimizar o sofrimento
\end{tabular}

12 Qualitativo/

Na vigência do câncer, a espiritualidade se apresenta como um apoio importante para construção dos significados da vida. A religiosidade pode aumentar o apoio social, porque provê acesso às redes sociais e estabelece formas de assistência, como cuidado espiritual nas fases de angústia aguda

13 Qualitativo/36 Os serviços religiosos são importantes na construção do sentido das possibilidades de cura a 75 anos para os pacientes oncológicos. Foi utilizada a entrevista em profundidade, nessa pesquisa

Fonte: elaboração dos autores, com base na revisão integrativa (2020). 